\title{
Gestación con éxito en paciente en programa de hemodiálisis. Revisión y recomendaciones
}

\author{
Blanca Aznar Buil, Carmen Llorente González, Lydia Moreno Zamora, María Sales Lamarca, Eva Vallés \\ Villagrasa, Pablo Iñigo Gil
}

Servicio de Hemodiálisis Hospital Clínico Universitario Lozano Blesa. Zaragoza. España

\begin{abstract}
Resumen
La gestación a término en una Unidad de Hemodiálisis es un proceso poco frecuente. Muchos factores colaboran a su baja incidencia; trastornos hormonales, psicológicos, complicaciones maternas y fetales. Hasta hace poco se ha desaconsejado e incluso contraindicado la gestación en estas fases de la Enfermedad Renal Crónica. En los últimos años se han dado recomendaciones para el manejo de esta situación de riesgo provocando controversia sobre el consejo que debemos de dar al respecto.
\end{abstract}

Presentamos un caso de una gestación a término en una paciente asiática de 27 años en nuestra Unidad de Hemodiálisis en la que el seguimiento de las recomendaciones condujo a un éxito en el desarrollo de la gestación y ausencia de complicaciones materno-infantiles. Estas pautas consistieron en un control estricto consistente en un incremento drástico de las horas de hemodiálisis semanal, uso de filtros de alta permeabilidad, control del peso y ganancia interdiálisis, tensión arterial, control ecográfico más frecuente, seguimiento multidisciplinar (nefrólogo, obstetra, enfermería) y dieta individualizada. Esta conducta puede cambiar significativamente el concepto de esta situación en nuestras mujeres en hemodiálisis en edad susceptible de gestación.

Nuestra experiencia en este caso ha sido positiva no presentando ninguna complicación tanto el recién nacido como la madre. En principio no se debería de descartar la lactancia materna.

\section{Correspondencia:}

Carmen Llorente González

Avda. Patio de los Naranjos, $263^{\circ}$ C. 50022 Zaragoza

E-mail: maryborjana@hotmail.com
PALABRAS CLAVE

- HEMODIÁLISIS

- EMBARAZO

- GESTACIÓN CON ÉXITO

- RECOMENDACIONES

Successful pregnancy in patients on hemodialysis. Review and Recommendations

\section{Abstract}

Successful pregnancy in a hemodialysis unit is a rare process. Many factors contribute to the low incidence; hormonal and psychological disorders, maternal and fetal complications. Until recently, pregnancy in these stages of chronic kidney disease has been discouraged and even contraindicated. In recent years there have been recommendations for the management of this risk provoking controversy about what the health professional must advise.

We report a case of a successful pregnancy in an 27-years Asian patient in our hemodialysis unit in which the monitoring of the recommendations led to a successful pregnancy and absence of maternal and child complications. These recommendations consisted of strict control leading in a drastic increase in hours of weekly hemodialysis, use of filters with high permeability, weight control and interdialysis gain, blood pressure, more frequent ultrasound control, multidisciplinary monitoring (nephrologist, obstetrician and nurse) and individualized diet. This behavior can significantly change the concept of this situation on women in hemodialysis with a susceptible gestational age.

Our experience in this case has been positive, without complications in both the mother and newborn. In principle, breastfeeding should not be ruled out. 


\section{KEYWORDS}

- HEMODIALYSIS

- SUCCESSFUL PREGNANCY

- GESTATION

- RECOMMENDATIONS

\section{Introducción}

El embarazo durante el tratamiento renal sustitutivo en Hemodiálisis (HD) es un hecho excepcional debido a varios factores. La frecuencia de embarazos a término es escasa, entre el $0.3-0.7 \%$ de las mujeres que están en HD en edad fértil ${ }^{1}$. Los datos existentes son insuficientes y probablemente incorrectos puesto que solo se publican los casos a término, se desconocen los abortos precoces y no hay registros sobre la verdadera situación de fertilidad de las pacientes.

Debido a la fisiopatología de la sexualidad en la Insuficiencia Renal Crónica (IRC), se sabe que existe amenorrea y anovulación en más del $50 \%$ de las pacientes en HD en edad fértil, hay aumento de la prolactina y de las hormonas $\mathrm{LH}$ y $\mathrm{FSH}$, lo cual produce alteraciones en el ciclo menstrual normal, alteración de las $\mathrm{GnRH}$, disminución de los estrógenos y por diversos factores también disminuye la libido y por tanto disminuye la frecuencia sexual ${ }^{2,3}$. Aun con todo en la IRC sigue siendo posible una concepción con éxito en cuyo caso existen múltiples factores que ponen en riesgo a la madre durante la gestación. Estos son: la hiperemesis ${ }^{4}$, que es ya un problema en un embarazo normal y empeora por la propia enfermedad renal poniendo en riesgo el estado nutricional que también esta ya comprometido en estos pacientes. La hipertensión, que aumenta considerablemente la posibilidad de preclampsia y eclamp$\mathrm{sia}^{5}$. Existe un peor manejo de la trombopenia y anemia gestacional ${ }^{6}$. Se corre el riesgo de de una isquemia placentaria debido a las hipotensiones intradiálisis. La dinámica del parto y postparto está alterada debido a las hemorragias. Se tiene un peor manejo de la colestasis gestacional. Mayor posibilidad de rotura de la bolsa amniótica debido al polihidramnios, lo que significa un importante riesgo de infección. Existe mayor riesgo de parto pretérmino. También se debe considerar el aumento del riesgo de alteración del acceso vascular al incrementar el número de sesiones 5 .

El feto no está exento de complicaciones puesto que hay mayor probabilidad de abortos, muchos de ellos no conocidos. En caso de llegar al final de la gestación existe aun así una elevada frecuencia de muerte neo- natal, en las primeras horas/días postparto y riesgo de parto prematuro. Pueden sufrir un retraso en el crecimiento intrauterino y existe una elevada frecuencia de bajo peso al nacer 4,5 , lo que puede desencadenar una serie de anormalidades congénitas relacionadas con trastornos neurológicos como epilepsia, ceguera, sordera, retraso madurativo ${ }^{7}$.

Las consecuencias del polihidramnios afectan también al feto. Estas pueden ser, además de la infección, desprendimiento precoz de la placenta, contracciones uterinas tempranas, mala posición fetal y accidentes con el cordón umbilical. El polihidramnios se debe probablemente a la diuresis de soluto fetal causada por el desmesurado aumento de la urea placentaria ${ }^{8}$. El niño también puede sufrir HTA, enfermedades cardiovasculares, distress respiratorio ${ }^{7}$.

Teniendo en cuenta la multitud de complicaciones y con el objetivo de evitarlas, al consultar la literatura se encuentran una serie de recomendaciones:

- Aumento del número de sesiones de HD, con el objetivo de controlar el metabolismo y la sobrecarga hídrica, y evitar que el embrión se encuentre en un ambiente urémico. Se recomienda incrementar las horas semanales hasta $20-24^{2,9}$. Esta modalidad permite optimizar el control de la ganancia de peso y de la TA. Por otro lado, permite prescribir una ingesta calórico y proteica materna adecuada a las necesidades del feto ${ }^{10}$. Se pretende mantener los niveles de uremia a menos de $100 \mathrm{mg} / \mathrm{dl}$.

- Usar membranas más biocompatibles para minimizar el estado inflamatorio

- Ajustar la heparinización, mantener niveles por encima de 50.000 plaquetas ${ }^{11}$.

- Control farmacológico, puesto que la gran mayoría de los medicamentos no están probados en embarazos, por lo que se tiene que valorar el riesgo-beneficio de mantenerlos o no, sobre todo en el primer tercio del embarazo y aquellos que sean previsiblemente teratogénicos ${ }^{12,13,14}$. En cada actuación se debe valorar la yatrogenia farmacológica y de técnicas diagnósticas.

\section{Caso clínico}

Mujer de 27 años de raza china, diagnosticada de IRC secundaria a enfermedad de Shönlein-Henoch controlada desde hacía años en consultas externas. Se diagnosticó de vasculitis leucocitoclástica por biopsia de 
piel y una glomerulonefrititis proliferativa mesangial con $42 \%$ de semilunas en la Biopsia Renal. Inició HD en agosto de 2009. Se inició estudio para inclusión en Lista de Espera de trasplante renal. En noviembre de
2009 se confirma embarazo. La Tabla 1 muestra los principales cambios llevados a cabo en la pauta de HD tras ser informados del Test positivo de embarazo de la paciente.

Tabla 1. Principales cambios en su rutina de HD.

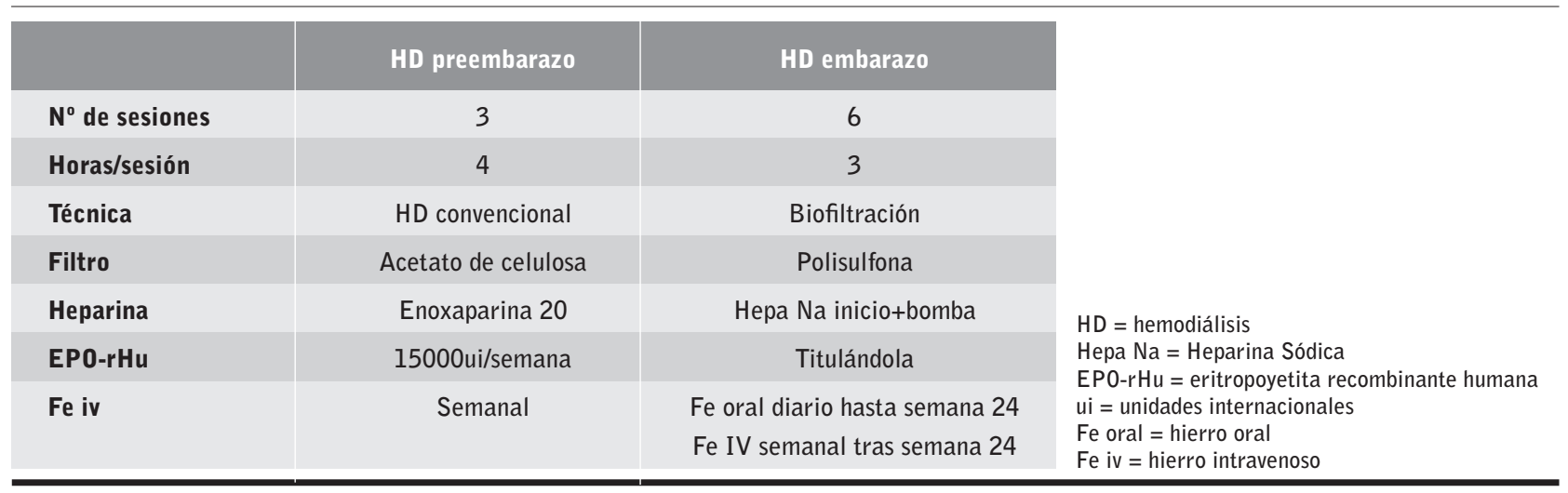

Inicialmente se suspendió el hierro iv., puesto que no está demostrada su seguridad y hay dudas sobre la toxicidad aguda del hierro en el feto. No obstante muchos grupos lo administran rutinariamente y lo que se aconseja es disminuir las dosis diarias ${ }^{15}$. En este caso fue sustituido por hierro oral, pero también hubo de ser reintroducido el intravenoso a dosis semanales tras la semana 24. Respecto a la eritropoyetina, su uso durante el embarazo ha demostrado ser seguro, no documentándose incremento de la presión sanguínea ni teratogenicidad $^{16}$. Reseñar que el tratamiento previo fue el que llevó hasta la semana 6 que es cuando se tiene conocimiento de la situación.
En la tabla 2 se muestran los datos evolutivos en el Peso y TA de la paciente a lo largo del embarazo. Se hizo un control exhaustivo de la TA. En la semana 8 se dio un aumento de ésta pero fue controlada manteniendo todo el embarazo TA sistólica alrededor de $120-130 \mathrm{mmHg}$. Valorado el riesgo, se optó por una tendencia a la hipertensión con el objetivo de disminuir las posibilidades de hipotensiones intradiálisis que tiene peores consecuencias ${ }^{12}$.

Tabla 2. Datos evolutivos de TA ( $\mathrm{mmHg}$ ) y Peso (Kgs).

\begin{tabular}{l|c|c|c|c|c|c|c|c|c|c|}
\hline & Inicio HD & 0 & 8 & 12 & 16 & 20 & 24 & 28 & 33 \\
\hline Peso $(\mathbf{k g})$ & 52 & 52 & 53 & 54 & 55.5 & 56 & 61 & 61.5 & 64.5 \\
\hline TAS & 130 & 123 & 152 & 140 & 130 & 120 & 130 & 130 & 120 & $\begin{array}{l}\text { HD = hemodiálisis } \\
\text { TAS = tensión arterial sistólica } \\
\text { TAD = tensión arterial diastólica }\end{array}$ \\
\hline TAD & 80 & 74 & 93 & 84 & 85 & 75 & 80 & 83 & 80 & TA \\
\hline
\end{tabular}

La paciente había iniciado HD hacía poco tiempo por ello el flujo fue aumentando progresivamente según la FAVI maduraba. La UF diaria fue de 0,6-0,71/h que, sumado a que conservaba una diuresis de 800 cc al día, indica que la paciente bebía abundante líquido. Otros parámetros se muestran en la Tabla 3.

Tabla 3. Datos evolutivos en HD.

\begin{tabular}{|c|c|c|c|c|c|c|c|c|c|c|}
\hline & Inicio HD & 0 & 8 & 12 & 16 & 20 & 24 & 28 & 33 & \multirow{4}{*}{$\begin{array}{l}\mathrm{Qb}(\mathrm{ml} / \mathrm{min})=\text { Flujo sanguíneo } \\
\text { (mililitros/minuto) } \\
\text { UF }(\mathrm{h})=\text { Ultrafiltración (hora) }\end{array}$} \\
\hline Ktv & 1.22 & 1.30 & 1.03 & 1.32 & 1.34 & 1.12 & 1.16 & 1.21 & 1.21 & \\
\hline$Q_{b}(\mathrm{ml} / \mathrm{min})$ & 310 & 300 & 330 & 380 & 380 & 350 & 380 & 360 & 385 & \\
\hline UF (h) & 0.58 & 0.72 & 0.69 & 0.63 & 0.69 & 0.5 & 0.770 & 0.72 & 0.59 & \\
\hline
\end{tabular}


Se realizaron numerosos controles ecográficos, (tabla 4) sobre todo en la última etapa donde se observa un polihidramnios que va en aumento a pesar de las medidas adoptadas.

Tabla 4. Parámetros embarazo, controles ecográficos.

\begin{tabular}{|c|c|c|}
\hline 9 semanas & 30 noviembre & Líquido amniótico normal \\
\hline 13 semanas & 29 diciembre & Líquido amniótico normal \\
\hline 21 semanas & 23 febrero & Líquido amniótico normal. Perímetro cefálico $189 \mathrm{~mm}$. Sexo varón \\
\hline 27 semanas & 08 abril & Hidramnios 20 puntos. Perímetro cefálico $254 \mathrm{~mm}$ \\
\hline 30 semanas & 29 abril & Hidramnios 40 puntos. Perímetro cefálico $277 \mathrm{~mm}$ \\
\hline 31 semanas & 05 mayo & $\begin{array}{l}\text { Contracciones. Ingreso. Valoración cesárea urgente } \\
\text { Tractocile para inhibir el parto y Esteroides para maduración pulmonar }\end{array}$ \\
\hline 32 semanas & 13 mayo & Hidramnios 45 puntos. Perímetro cefálico 308mm \\
\hline \multirow[t]{2}{*}{33 semanas } & 24 mayo & $\begin{array}{l}\text { 9:30 Se indica cesárea urgente. Polihidramnios } 2,5-2,8 \text { litros. } \\
\text { Peso placenta } 540 \mathrm{~g} \text {. Recién nacido } 2250 \mathrm{~g} \text { sin malformaciones. } \\
\text { Se realiza HD a última hora a la madre }\end{array}$ \\
\hline & 30 Mayo & Alta tras 6 días \\
\hline
\end{tabular}

Ingresa en la semana 31 con contracciones. Se valora cesárea urgente por presentación podálica y se utiliza el tractocile para intentar inhibir el parto y esteroides para maduración pulmonar. Se consigue retrasar el parto casi tres emanas mas, indicando entonces cesárea urgente. Se cuantificó un polihidramnios entre 2,5-2,8 litros. La placenta pesó $540 \mathrm{~g}$. El niño pesó $2.250 \mathrm{~g}$.

Ese mismo día se retomaron las diálisis de la madre volviendo a las pautas anteriores al embarazo, y recibe el alta hospitalaria seis días más tarde.

El niño fue ingresado en la UCI neonatal las primeras 24 horas a modo de prevención. No presentó malformaciones, con un test de APGAR de 9 en el primer minuto y de 9 en el quinto. Disfrutó de lactancia mixta. En la analítica se pudo observar en el niño una creatinina elevada de $4,5 \mathrm{mg} / \mathrm{dl}$ al nacer que por sus propios medios corrige a 0,7 tras 24 horas.

La revisión del puerperio se realizó un mes y medio más tarde, siendo todos los parámetros normales y destacando que la paciente vuelve a tener la menstruación en ese momento.

\section{Discusión}

Presentamos un caso de embarazo a término sin complicaciones de una paciente con ERC en HD. Tanto la madre como el niño están en perfecto estado y la evolución ha sido satisfactoria.
Hacemos hincapié en los cuidados que enfermería ha de desarrollar para colaborar en el buen término del proceso:

- Apoyo emocional, ya que, a las alteraciones hormonales de una gestante, se añade el miedo a lo que les pueda suceder a ambos, además de que ha de acudir a diario a sus sesiones de hemodiálisis.

- Estricto control del balance hídrico, para lo que se le recomendó que registrara diariamente la ingesta de líquido y la diuresis.

- También es importante tener en cuenta la ganancia de peso propia de la gestación.

- Especial cuidado de la fístula que, a pesar de ser relativamente nueva y de las punciones diarias, no presentó complicaciones.

- Controles analíticos semanales.

- Pautas dietéticas teniendo en cuenta la cultura y situación de la paciente.

- Estrecha relación interdisciplinar.

- Control exhaustivo en las sesiones de hemodiálisis por la multitud de complicaciones que podían surgir.

- Control de la ansiedad, sobre todo al final del embarazo.

- Fomento de la relación madre-hijo considerando la posible depresión post parto y que el niño tenga que ingresar en una UCI neonatal.

Con el objetivo principal de prevenir estados de malnutrición, resaltar que ha habido importantes cambios 
en las pautas, pasando de muy restrictivas en el pasado a la tendencia actual de dejar dietas más libres². En ocasiones se opta incluso por administrar nutrición enteral adicional durante las sesiones de HD para cubrir carencias ${ }^{4}$.

En nuestro caso, por su cultura, llevó una dieta basada fundamentalmente en arroz. Esta dieta ya fue descrita como optima para pacientes renales en los 60 . La base de esta dieta erradica en la riqueza en aminoácidos esenciales siendo baja en proteínas totales, dando como resultado una menor producción de urea y un aumento del apetito ${ }^{17}$. Apoyando esta teoría, está demostrado un aumento de la supervivencia neonatal, mayor peso al nacer y mayor edad gestacional con niveles de nitrógeno urémico (BUN) prediálisis menores a $50 \mathrm{mg} / 100 \mathrm{ml}^{16}$. Se le recomendó no obstante aumentar la cantidad de otros alimentos siguiendo una dieta libre. No se restringió la cantidad de líquidos ya que mantenía una diuresis residual importante y no hubo necesidad de aportes nutricionales extra.

El polihidramnios en este caso es debido no tanto a la sobrecarga hídrica, sino que es consecuencia de la diuresis de soluto fetal causada por el aumento de la urea placentaria ${ }^{8}$ por lo que resulta más complicado su control.

Sobre la lactancia materna, hemos de señalar, que históricamente se retiraba por precaución, más bien debido a la escasa experiencia profesional ${ }^{18}$. Consultado con el comité de lactancia de la AEP (Asociación Española de Pediatría): "No hay razones justificadas para retirar la lactancia en caso de diálisis. El riesgo de hepatitis $C$ es mayor entre personas que se dializan, pero la hepatitis $C$ no contraindica la lactancia. La lactancia no tiene por qué afectar al balance de líquidos y solutos que se realizan en diálisis". En este caso, el niño disfruto de lactancia mixta debido a que la hermana de la madre dio a luz el día anterior y dio lactancia en tándem a los dos niños hasta los 5 meses.

Considerada la bibliografía cabe ser optimistas dado que la tasa de éxito ha pasado de ser del $23 \%$ en los años 80 a más del $70 \%$ para el año 2008 teniendo en cuenta que en la población general es del $95 \%$, por lo que se está planteando cambiar la política de consejo en las pacientes en tratamiento con HD sobre este tema ${ }^{19}$. De hecho hay quien plantea que posponer el embarazo hasta que se reciba un trasplante de riñón aunque sea lo ideal, puede llevar años y se corre el riesgo de que la ERC se convierta en lo suficientemente grave como para requerir un trasplante, puede haber una larga es- pera para recibir un órgano de donante adecuado, por lo que las mujeres podrían ser capaces de recibir un trasplante de riñón cuando se les hayan pasado sus años reproductivos ${ }^{20}$. No obstante hay que ser cautelosos ya que se deben considerar múltiples factores como que la posibilidad de embarazo con éxito depende del diagnóstico por el cual están en situación de IRC. Así mismo influye el tiempo que hace que están en programa renal sustitutivo, siendo más exitosos aquellos en los que las mujeres habían empezado hacía poco la diálisis e incluso las que debutan durante el embarazo frente a aquellas que llevan años en diálisis ${ }^{20}$. También parece ser menos peligroso un embarazo en programa de hemodiálisis que aquellos que tienen lugar en las etapas previas a la insuficiencia renal terminal ${ }^{21}$.

\section{Conflicto de intereses}

Los autores declaran estar libres de cualquier asociación personal o comercial que pueda suponer un conflicto de interés.

\section{Recibido: 8 abril 2015 \\ Revisado: 10 junio 2015 \\ Modificado: 2 septiembre 2015 \\ Aceptado: 12 septiembre 2015}

\section{Bibliografía}

1. López Menchero R, Albero MD, Cabeza B, Álvarez L, Del Pozo C, Sánchez L. Successful pregnancy in a patient with systemic lupus erythematosus on hemodialysis. Nefrologia. 2004; 24(1):70-4.

2. Giatras I, Levy DP, Malone FD, Carlson JA, Jungers P. Pregnancy during dialysis: case report and management guidelines. Nephrol Dial Transplant. 1998; 13(12):3266-72.

3. Farreras Rozman. Insuficiencia renal crónica avanzada o terminal: síndrome urémico: trastornos endocrinos. Medicina interna Volumen II. Madrid: Decimotercera edición. Editorial Mosby; 1995. p. 891-892. 
4. Tuot $D$, Gibson $S$, Caughey $A B$, Frassetto LA. Intradialytic hyperalimentation as adjuvant support in pregnant hemodialysis patients: case report and review of the literature. Int Urol Nephrol. 2010; 42(1):233-7.

5. Bolignano D, Coppolino G, Crasci E, Campo S, Aloisi C, Buemi M. Pregnancy in uremic patients: An eventful journey. J. Obstet. Gynaecol. 2008; 34(2):137-143.

6. Cararach V, Almirall J, Heredia L, Usatorre $T$, Hemodiálisis periódica y gestación con feto vivo. Clin Invest Ginecol Obstet. 1988; 15(3):119-123.

7. Hershkovitz Zui Burbea D, Shorecki K, Brenner B. Fetal programming of adult kidney disease: cellular and molecular mechanisms. Clin J Am Soc Nephrol. 2007; 2(2):334-342.

8. Bernasconi $A R$, Lapidus $A M$, Waisman $R$, Liste $A A$, Voto LS, Heguilen RM, Diálisis y embarazo, 13 años de experiencia en el hospital público. Revista de Nefrología, Diálisis y Trasplante 2007; 27(3): 103-108.

9. Hladunewich MA et al. Intensive Hemodialysis associates with improved pregnancy outcomes: A Canadian and United States cohort comparison. J Am Soc Nephrol. 2014; 25(5):1103-1109.

10. Holley JL. Reddy SS. Pregnancy in Dialysis patients a review of outcomes, complications, and management. Semin. Dial 2003; 16(5):384-8.

11. Muñoz García VU, Vaca Ruiz AM, García Criado J, Mohamed Maanan N, Rosa Fortes I, García Salas V. Gestación con éxito en una paciente con anemia y plaquetopenia en programa de hemodiálisis a través de catéter. Revista de la Sociedad Española de Enfermería Nefrológica 2009; 12(1).

12. Carretero Sierra C. et. al. Gestación con éxito en paciente con hipotiroidismo en programa de diálisis. Revista de la Sociedad Española de Enfermería Nefrológica. 2006; 9(4).
13. Tan LK, Kanagalingam D, Tan K, Choong H.L Obstetrics outcomes in women with end-stage renal failure requiring dialysis. Int $\mathrm{J}$ Gynaecol Obstet, 2006; 94(1):17-22.

14. Hou S, Orlowski J, Palhl M, Ambrose S, Hussey $M$, Wong $D$, Pregnancy in women with end-stage renal disease: treatment of anemia and premature labor. Am J. Kidney Dis 1993; 21(1): 16-22.

15. Vallejos A. Embarazo en diálisis. Revista de Nefrología, Diálisis y Trasplante 2004; 24(4):171178.

16. Karina R. et al. Embarazo en mujeres en diálisis crónica: Revisión. Nefrología 2012;32 (3).

17. Giovanetti S, Maggiore Q. A low nitrogen diet with proteins of high biological value for severe chronic uraemia. Lancet, 1964; 9;1(7341):1000-3.

18. Lindhemimer MD, Davison JM, Katz AI. The kidney and hypertension in pregnancy: twenty exiting years. Semin Nephrol 2001; 21: 173-89

19. Piccoli GB et al. Pregnancy in dialysis patients: Is the evidence strong enough to lead us to change our counseling policy? Clin J Am Soc Nephrol 2010; 5: 62-71.

20. Jesudason S, Grace BS, Stephen P. McDonald. Pregnancy outcomes according to dialysis commencing before or after conception in women with ESRC. Clin J Am Soc Nephrol. 2014 Jan 7; 9(1): 143-149.

21. Castellano G, Losappio V, Gesualdo L. Update on pregnancy in chronic kidney disease. Kidney Blood Press Res 2011; 34(4):253-60. 